\title{
Mutant studies of phosphofructo-2-kinases do not reveal an essential role of fructose-2,6- bisphosphate in the regulation of carbon fluxes in yeast cells
}

\author{
Susanne Müller, Friedrich K. Zimmermann and Eckhard Boles†
}

Institut für Mikrobiologie und Genetik, Technische Hochschule Darmstadt, Schnittspahnstr. 10, D64287 Darmstadt, Germany

\author{
Author for correspondence: Eckhard Boles. Tel: +49 21181 12778. Fax: +49 2118115370 \\ e-mail: boles@uni-duesseldorf.de
}

\begin{abstract}
The effect of the allosteric regulator fructose-2,6-bisphosphate (F2,6bP) on the regulation of carbohydrate metabolism was investigated in vivo with Saccharomyces cerevisiae mutants containing no, very high or unregulated 6phosphofructo-2-kinase activity. Simultaneous overproduction of F2,6bP and 6phosphofructo-1-kinase activity did not increase the glycolytic flux to ethanol. Overexpression of fructose-1,6-bisphosphatase during growth on glucose in a mutant strain devoid of F2,6bP did not cause pronounced effects on the cells. Moreover, high levels of $\mathbf{F 2 , 6 b P}$ during growth on ethanol in a strain with a highly active 6-phosphofructo-2-kinase enzyme did not affect either carbon flux to glycogen or growth rate. Site-directed mutagenesis of 6-phosphofructo2-kinase (Pfk26) revealed that serine 644 is involved in the activation of Pfk26 by protein kinase A phosphorylation, but that, additionally, the enzyme can be further activated by phosphorylation of another amino acid residue. The results demonstrate that $F 2,6 b P$ is not needed to sustain an adequate glycolytic flux under fermentative conditions, but rather is concerned with the homeostasis of metabolite concentrations. Moreover, they fail to indicate a physiological significance for inhibition of fructose-1,6-bisphosphatase by F2,6bP.
\end{abstract}

Keywords: Saccharomyces cerevisiae, fructose-2,6-bisphosphate, 6-phosphofructo-1kinase, glycolytic flux, futile cycling

\section{INTRODUCTION}

Since its discovery fructose-2,6-bisphosphate (F2,6bP) has been proposed to be the predominant effector of carbohydrate metabolism in Saccharomyces cerevisiae. Yeast 6-phosphofructo-1-kinase (6PF-1-K) (EC 2.7.1.11) is activated and fructose-1,6-bisphosphatase (F-1,6-bPase) (EC 3.1.3.11) is inhibited in vitro by nanomolar concentrations of F2,6bP (Lederer et al., 1981 ; Bartrons et al., 1982; Noda et al., 1984; François et al., 1987; Van Schaftingen, 1987). In yeast cells

\footnotetext{
Abbreviations: F2,6bP, fructose-2,6-bisphosphate; 6PF-1-K, 6phosphofructose-1-kinase; F-1,6-bPase, fructose-1,6-bisphosphatase; F6P, fructose-6-phosphate; $F 1,6 \mathrm{bP}$, fructose-1,6-bisphosphate; 6PF-2-K, 6phosphofructo-2-kinase.

†Present address: Institut für Mikrobiologie, Universităt Düsseldorf, Universitătsstr. 1, Geb. 26.12.01, D-40225 Dusseldorf, Germany
}

growing on respiratory carbon sources (gluconeogenic conditions), F2,6bP concentrations are low but increase rapidly after the addition of fermentable sugars. Therefore, $\mathrm{F} 2,6 \mathrm{bP}$ is thought to be crucial for the activation of glycolysis and to be a main determinant in preventing the energy-wasting cycling between fructose-6-phosphate (F6P) and fructose-1,6-bisphosphate (F1,6bP) (Hers \& Van Schaftingen, 1982; François et al., 1987, 1988; Navas et al., 1993).

In yeast cells, two isoenzymes of 6-phosphofructo-2kinase (6PF-2-K) (EC 2.7.1.105) catalyse the synthesis of F2,6bP from F6P and ATP. One of these isoenzymes, encoded by the gene PFK26 (Kretschmer \& Fraenkel, 1991), is activated by protein kinase A phosphorylation, while synthesis of the second isoenzyme, encoded by the gene PFK27 (Boles et al., 1996), is induced by fermentable carbon sources. F2,6bP is degraded by a specific fructose-2,6-bisphosphatase, encoded by the 
gene FBP26 (Paravicini \& Kretschmer, 1992), and by unspecific phosphatases.

Recently, it has been demonstrated that a yeast $p f k 26$ $p f k 27$ double-deletion mutant strain that is unable to synthesize F2,6bP (Boles et al., 1996) and a yeast mutant strain containing a $6 \mathrm{PF}-1-\mathrm{K}$ insensitive to $\mathrm{F} 2,6 \mathrm{bP}$ (Heinisch et al., 1996) are able to grow normally on fermentable sugars and have flux rates for glucose utilization and ethanol production similar to a wild-type strain. These results question the role of $F 2,6 \mathrm{bP}$ as an important determinant of glycolytic flux regulation in $S$. cerevisiae. To further investigate the physiological relevance of $F 2,6 \mathrm{bP}$ in yeast carbon metabolism, we have constructed mutants affected in the amount and the control of 6PF-2-K, 6PF-1-K and F-1,6-bPase activity. Investigations with these mutant strains confirm that the level of F2,6bP does not regulate glycolytic flux rates during glucose fermentation and fail to indicate a major role for $\mathrm{F} 2,6 \mathrm{bP}$ in the prevention of futile cycling of the 6PF-1-K/F-1,6-bPase enzyme pair.

\section{METHODS}

Strains, media and growth conditions. All yeast strains used in this study were derived from strains VW-1A (MATa leu23,112 ura3-52 trp1-289 his3- 1 MAL2-8 ${ }^{\circ}$ SUC2), VW-1B

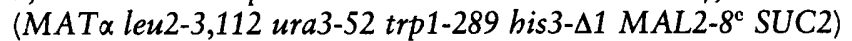
or the corresponding diploid strain (VW-1D) unless otherwise stated. The strains CEN.PK113-9D (MATa LEU2 ura3-52 trp1-289 HIS3 MAL2-8 SUC2) and CEN.PK113-3C (MATa LEU2 URA3 trp1-289 HIS3 MAL2-8 ${ }^{\circ}$ SUC2) (kindly provided by $P$. Kötter, Institut für Mikrobiologie, Frankfurt, Germany) are isogenic to strain VW-1A. Strains VW.EB-3A ( $p f k 26 \Delta)$, VW.EB-9A and 9B ( $p f k 1 \Delta p f k 26 \Delta)$, and VW.EB-13A and 13B ( $p f k 26 \Delta p f k 27 \Delta$ ) were described previously (Boles et al., 1996). Yeast cells were grown at $28^{\circ} \mathrm{C}$ in batch cultures as described by Boles et al. (1996). Escherichia coli strains JM101 and SURE (Stratagene) were used for the propagation of plasmids.

Molecular biology techniques. DNA was prepared and manipulated as described by Sambrook et al. (1989). Yeastspecific techniques were as described by Guthrie \& Fink (1991). DNA was transformed into yeast according to Schiestl \& Gietz (1989) and in vitro mutagenesis was as described by Boles \& Miosga (1995).

Construction of plasmids and mutant strains. Plasmid pAN11 containing the $F B P 1$ gene behind the $A D H 1$ promoter, plasmid pPFK-D1.2 containing the genes PFK1 and PFK2, and plasmid pMK2 containing the PFK 26 gene were described by Navas $e t$ al. (1993), Schaaff et al. (1989), and Kretschmer et al. (1991), respectively. The wild-type and different mutant PFK26 alleles obtained after in vitro mutagenesis and sequencing (Boles \& Miosga, 1995) were recloned into YIplac211 (Gietz \& Sugino, 1988), linearized with EcoRV and, after transformation, integrated once or severalfold, [ ] $]_{x}$, into the ura 3 locus of strain VW.EB-13A ( $p f k 26 \Delta p f k 27 \Delta$ ), resulting in strains VW.EB-14B $\left(\right.$ Pfk26 $\left.{ }^{\text {Ser644 }}\right)$ VW.EB-14C (Pfk26 $\left.{ }^{\text {Ala644 }}\right)$, VW.EB-14D $\left(\mathrm{Pfk} 26^{\mathrm{Asp644}}\right)$, and VW.EB-14E ([Pfk26 $\left.\left.{ }^{\mathrm{Asp644}}\right]_{x}\right)$. An EcoRI/SalI digest of plasmid pIJdel2 (Boles et al., 1993) was used to replace the majority of the PFK2 gene in the strain VW-1B with the yeast $U R A 3$ gene, resulting in strain VW.EB-2B $(p f k 2 \Delta)$. This strain was crossed with strain VW.EB-3A $(p f k 26 \Delta)$, the diploid strain was sporulated and the tetrads dissected, resulting in the $p f k 2 p f k 26$ double-deletion mutant

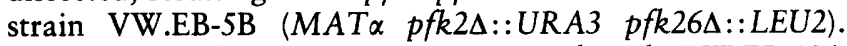
VW.EB-5B and VW.EB-9B were crossed with VW.EB-13A and after sporulation they were used to construct the $p f k 1$ $p f k 26 p f k 27$ and $p f k 2 p f k 26 p f k 27$ triple-deletion mutants.

Determination of enzyme activities and metabolite levels. Crude extracts of the cells were prepared according to Ciriacy \& Breitenbach (1979). 6PF-2-K activity was assayed according to Kretschmer et al. (1991). F2,6bP concentrations were determined after 0,3 and $5 \mathrm{~min}$ with pyrophosphate-dependent fructose-6-phosphate kinase from potato tuber (Sigma) as described by François et al. (1984). F-1,6-bP activity was determined according to Gancedo \& Gancedo (1971) in $50 \mathrm{mM}$ imidazole buffer $\mathrm{pH} 7 \cdot 0$, containing $10 \mathrm{mM} \mathrm{MgCl}$, $100 \mathrm{mM} \mathrm{KCl}$ and $0.1 \mathrm{mM}$ EDTA. 6PF-1-K activity was determined as described by Heinisch et al. (1989). The protein concentrations were determined by the microbiuret method (Zamenhoff, 1957) using bovine serum albumin as a standard. Metabolite extracts were prepared as described by Boles \& Zimmermann (1993). F2,6bP concentrations were determined according to Van Schaftingen et al. (1982) and the concentrations of glycolytic metabolites were assayed enzymically (Bergmeyer, 1974). Yeast cultures to be used for glycogen determination were chilled quickly by addition of an equal volume of a methanol and $\mathrm{H}_{2} \mathrm{O}$ mixture $(1: 1)$ at $-50^{\circ} \mathrm{C}$. Cells were collected by centrifugation at $4^{\circ} \mathrm{C}$ and washed three times with distilled water at $4^{\circ} \mathrm{C}$. Glycogen was determined after nitrogen starvation as described by Lillie $\&$ Pringle (1980). The experiments were repeated at least once, with similar results.

Determination of growth rates, glucose consumption and ethanol production. A Beckman DU-40 spectrophotometer and $10 \mathrm{~mm}$ cuvettes were used for all spectrophotometric analyses. Growth rate, glucose consumption and ethanol production were determined as described previously (Boles et al., 1996). The experiments were repeated at least once, with similar results.

\section{RESULTS AND DISCUSSION}

\section{Increasing both F2,6bP concentrations and 6PF-1-K activity does not accelerate glycolytic flux}

Although it has been thought for a long time that 6PF-1$\mathrm{K}$ is the rate-limiting step of glycolysis, a more than threefold overexpression of 6PF-1-K did not cause an effect on the anaerobic glycolytic flux to ethanol (Heinisch, 1986; Schaaff et al., 1989). However, Davies \& Brindle (1992) observed that yeast cells overexpressing 6PF-1-K showed a compensatory decrease in the concentration of $\mathrm{F} 2,6 \mathrm{bP}$, which is a potent activator of $6 \mathrm{PF}-$ $1-K$. They proposed that this decrease could significantly lower the activity of 6PF-1-K in vivo and compensate for the increased amount of 6PF-1-K. Nevertheless, it has been demonstrated recently (Boles et al., 1996) that a yeast mutant strain unable to synthesize F2,6bP had flux rates for glucose utilization and ethanol production similar to those of the wild-type strain, indicating that $\mathrm{F} 2,6 \mathrm{bP}$ is dispensable in vivo for the activation of $6 \mathrm{PF}-1$ $\mathrm{K}$. To further investigate the role of $\mathrm{F} 2,6 \mathrm{bP}$ in the regulation of glycolytic flux, we constructed a yeast strain that simultaneously overproduced $\mathrm{F} 2,6 \mathrm{bP}$ and overexpressed 6PF-1-K. Plasmid YIplac-PFK26 ${ }^{\text {Asp644 }}$ 
Table 1. Glucose-consumption rates and ethanol-production rates of the wild-type ( $\mathrm{WW}$ $1 \mathrm{~A}$ ) and a mutant strain overproducing F2,6bP and 6PF-1-K (UW.SM-2A)

The cultures were pre-grown to the mid-exponential phase in synthetic complete medium with $2 \%$ glucose lacking leucine, and then washed once with and transferred to the same pre-warmed medium. The data presented are the means of two or three different experiments \pm deviation from the mean or $\pm S D$, respectively (also applies to Tables 2 and 3 ).

\begin{tabular}{|c|c|c|c|c|c|}
\hline Strain & Genotype & F2,6bP* & 6PF-1-K† & $V_{\mathrm{Glc}} \ddagger$ & $\boldsymbol{P}_{\text {ErOH }} \S$ \\
\hline VW-1A & Wild-type & $7 \cdot 5 \pm 1 \cdot 0$ & $240 \pm 30$ & $5 \cdot 1 \pm 0 \cdot 5$ & $8.0 \pm 0.7$ \\
\hline VW.SM-2A & YIplac- $P F K 26^{\text {Asp644 }}$ pPFK-D1.2 & $17 \cdot 4 \pm 1 \cdot 9$ & $650 \pm 50$ & $4.8 \pm 0.5$ & $7 \cdot 6 \pm 0.6$ \\
\hline
\end{tabular}

${ }^{*} \mathrm{~F} 2,6 \mathrm{bP}$ concentration in pmol (mg dry weight) ${ }^{-1}$.

$\dagger 6 \mathrm{PF}-1-\mathrm{K}$ activity in $\mathrm{nmol} \mathrm{min}^{-1}$ (mg protein) ${ }^{-1}$.

‡Glucose-consumption rate in $\mathrm{mM} \mathrm{h}^{-1}$ per $\mathrm{OD}_{600}$ unit.

\Ethanol-production rate in $\mathrm{mM} \mathrm{h}^{-1}$ per $\mathrm{OD}_{600}$ unit.

encoding a constitutively activated Pfk $26^{\text {Asp644 }}$ enzyme (see below) was integrated severalfold into the genome of the wild-type strain VW-1A. A transformant producing high concentrations of F2,6bP was selected and transformed with multi-copy plasmid pPFK-D1.2 containing the two genes encoding the subunits of $6 \mathrm{PF}-1-\mathrm{K}$. Both the concentration of $\mathrm{F} 2,6 \mathrm{bP}$ and the $6 \mathrm{PF}-1-\mathrm{K}$ activity in this strain (VW.SM-2A) when grown in a synthetic medium with $2 \%$ glucose were elevated twoto threefold as compared with the corresponding wildtype strain VW-1A (Table 1). Nevertheless, glucoseconsumption and ethanol-production rates of this strain were identical to the wild-type strain (Table 1), indicating that neither the concentration of $F 2,6 \mathrm{bP}$ nor the activity of $6 \mathrm{PF}-1-\mathrm{K}$ was the rate-limiting factor of glycolytic flux.

These results together with our previous observations (Boles et al., 1996) undermine the traditional role of the allosteric effector $\mathrm{F} 2,6 \mathrm{bP}$ as a regulator of metabolic flux. Instead, they support the model that allosteric effects are more likely to be concerned with homeostasis of metabolite concentrations than with the control of metabolic fluxes (Fell \& Thomas, 1995; Thomas \& Fell, 1996).

\section{The Pfk2 but not the Pfk1 subunit of 6PF-1-K is sensitive to the absence of $F 2,6 b P$}

In contrast to $p f k 1 p f k 2$ double mutants, which do not grow on glucose medium, $p f k 1$ or $p f k 2$ single mutants grew well on all kinds and concentrations of carbon sources, although no 6PF-1-K activity could be detected in crude extracts of these cells (Breitenbach-Schmitt et al., 1984). It has been proposed that each of the subunits can serve both catalytic and regulatory functions (Arvanitidis \& Heinisch, 1994; Heinisch et al., 1996). To test whether both subunits are also equivalent with respect to their susceptibility to $\mathrm{F} 2,6 \mathrm{bP}, p f k 1 p f k 26$ $p f k 27$ and $p f k 2 p f k 26 p f k 27$ triple-deletion strains were constructed by crossing strain VW.EB-13A ( $p f k 26$ $p f k 27)$ with strains VW.EB-9B ( $p f k 1 p f k 26)$ and VW.EB-
5B ( $p f k 2 p f k 26)$, respectively, followed by sporulation and tetrad dissection. This analysis revealed that mutant cells containing only the PFK2-encoded subunit of $6 \mathrm{PF}$ $1-\mathrm{K}$ but unable to produce $\mathrm{F} 2,6 \mathrm{bP}$ ( $p f k 1 p f k 26 p f k 27$ ) no longer grew on a glucose-containing medium but did grow on an ethanol-containing medium, indicating that the Pfk2 subunit alone is not active or not stable in the absence of F2,6bP. In contrast, the PFK1-encoded subunit is not sensitive to the absence of $F 2,6 \mathrm{bP}$ as the growth rates of $p f k 2 p f k 26 p f k 27$ triple mutants on $0 \cdot 1-2 \%$ glucose-containing media were indistinguishable from $p f k 2$ single mutants. These results indicate that although both the two 6PF-1-K subunits can serve catalytic and regulatory functions, the subunits are not completely equivalent.

\section{The absence of $F 2,6 b P$ in yeast cells overexpressing F-1,6-bPase does not have deleterious effects on the cells}

$\mathrm{F} 2,6 \mathrm{bP}$ is a strong inhibitor of the gluconeogenic enzyme F-1,6-bPase in vitro, (Gancedo et al., 1982; Noda et al., 1984). It has been argued that this very strong inhibition of F-1,6-bPase by F2,6bP is responsible for the prevention of futile cycling between $\mathrm{F} 6 \mathrm{P}$ and $\mathrm{F} 1,6 \mathrm{bP}$ in a strain expressing high levels of gluconeogenic F-1,6bPase during growth on glucose (Navas et al., 1993). Interestingly, an increase in glucose utilization and ethanol production of $10-20 \%$ could be observed in this strain.

We investigated whether high-level expression of F-1,6bPase under glycolytic growth conditions in a $p f k 26$ pfk27 mutant strain without F2,6bP would impair growth and ethanol production due to the expected futile cycling or whether it was possible to increase glycolytic flux even more under such conditions. The multi-copy plasmid pAN11 (Navas et al., 1993), carrying the yeast $F B P 1$ gene (encoding F-1,6-bPase) controlled by the strong $A D H 1$ promoter was transformed into the $p f k 26 p f k 27$ double mutant. However, no significant differences in the generation times on glucose media between this strain and the corresponding wild-type 
Table 2. Growth rate, ethanol-production rate, F-1,6-bPase activity and concentrations of F6P and F1,6bP in the wild-type strain CEN.PK113-3C, in CEN.PK113-9D transformed with pAN11, and in the corresponding pfk26 pfk27 double-deletion strain

Cultures of the wild-type strain CEN.PK113-3C, this strain transformed with pAN11 (containing the FBP1 gene behind the $A D H 1$ promoter), and the corresponding $p f k 26 p f k 27$ double-deletion strain VW.EB-13A, lacking F2,6bP, were pre-grown to the mid-exponential phase in synthetic complete medium with $2 \%$ glucose lacking uracil, leucine and histidine, and then washed once with and transferred to the same pre-warmed medium. See Table 1 for details of reproducibility.

\begin{tabular}{|c|c|c|c|c|c|c|}
\hline Strain & Plasmid & $\boldsymbol{P}_{\mathrm{ErOH}}{ }^{*}$ & F-1,6-bPase $\dagger$ & $\mu \neq$ & F6PS & F1,6 bPS \\
\hline $\begin{array}{l}\text { CEN.PK113-3C } \\
\text { (wild-type) }\end{array}$ & - & $12 \cdot 6 \pm 0 \cdot 4$ & $<2$ & $0.38 \pm 0.02$ & $2 \cdot 5 \pm 0 \cdot 1$ & $4 \cdot 6 \pm 0 \cdot 1$ \\
\hline $\begin{array}{l}\text { CEN.PK113-9D } \\
\text { (wild-type) }\end{array}$ & $\begin{array}{c}A D H 1-F B P 1 \\
\text { (pAN11) }\end{array}$ & $14 \cdot 3 \pm 0 \cdot 4$ & $190 \pm 10$ & $0 \cdot 41 \pm 0 \cdot 01$ & $3 \cdot 3 \pm 0 \cdot 2$ & $5 \cdot 7 \pm 0 \cdot 1$ \\
\hline $\begin{array}{l}\text { VW.EB-13A } \\
(p f k 26 p f k 27)\end{array}$ & $\begin{array}{c}A D H 1-F B P 1 \\
\text { (pAN11) }\end{array}$ & $12 \cdot 6 \pm 0 \cdot 5$ & $230 \pm 10$ & $0.39 \pm 0.01$ & $5 \cdot 4 \pm 0 \cdot 2$ & $2 \cdot 6 \pm 0 \cdot 3$ \\
\hline
\end{tabular}

*Ethanol-production rate in $\mathrm{mM} \mathrm{h}^{-1}$ per $\mathrm{OD}_{600}$ unit.

† F-1,6-bPase activity in $\mathrm{nmol} \mathrm{min}^{-1}$ (mg protein) ${ }^{-1}$.

$\ddagger$ Maximum specific growth rate, $\mu_{\max }\left(\mathrm{h}^{-1}\right)$.

\$F6P and F1,6bP concentrations in nmol (mg dry weight) ${ }^{-1}$.

strains were observed (Table 2), indicating either that F2,6bP inhibition of F-1,6-bPase is not essential for the growth and survival of the cells or that other inhibitors such as AMP effectively substitute for F2,6bP (Noda et al., 1984). Moreover, it has been shown recently that the overexpression in yeast cells of a nearly uncontrolled bacterial mutant F-1,6-bPase caused cycling between F6P and F1,6bP of about $14 \%$ but did not cause deleterious effects to the yeast cells (Navas \& Gancedo, 1996).

Nevertheless, the increase in the ethanol-production rate of the wild-type strain overexpressing F-1,6-bPase returned to normal levels in the mutant cells without F2,6bP and simultaneously overexpressing F-1,6-bPase (Table 2). This suggests that, at least under these specific conditions, activation of $6 \mathrm{PF}-1-\mathrm{K}$ by F2,6bP is necessary to enhance glycolytic flux. Alternatively, it could be speculated that in the absence of F2,6bP inhibition the F1,6-bPase contribution to a futile cycle is too high so flux is decreased back to the wild-type rate. Interestingly, the concentration of F6P was very high and that of F1,6bP was low (Table 2). This has already been observed in $p f k 26$ pfk27 double mutant cells not overexpressing F-1,6-bPase (Boles et al., 1996). Therefore, the latter explanation is not very likely as it has been shown that a high rate of futile cycling in fact increases the fermentation rate (Navas \& Gancedo, 1996).

\section{Regulation of 6PF-2-K activity}

It has been described that Pfk 26 is activated by fermentable sugars via the RAS-adenylate cyclase signalling pathway (François et al., 1984; Kretschmer \& Fraenkel, 1991; Thevelein, 1991). The serine residue at amino acid position 644 of Pfk 26 has been thought to be phosphorylated by protein kinase A after glucose addition, leading to activation of the enzyme (Kretschmer \& Fraenkel, 1991). To test this hypothesis, mutant alleles of PFK26 at this position were constructed by site-directed mutagenesis as described by Boles $\&$ Miosga (1995). One of these alleles contained an alanine at amino acid position 644 ( $\mathrm{Pfk} 26^{\text {Ala644}}$ ) instead of the wild-type serine residue. Another allele contained an aspartate at position 644 (Pfk26 $\left.{ }^{\mathrm{Asp644}}\right)$. An alanine residue can no longer be phosphorylated by protein kinase A, and aspartate has been shown in some cases to mimic a phosphorylated serine residue (Marcus et al., 1988). The wild-type and mutant alleles were stably integrated into the genome of the $p f k 26 p f k 27$ doubledeletion strain. In the strain carrying the wild-type allele, 6PF-2-K activity increased about fourfold $10 \mathrm{~min}$ after the addition of glucose to ethanol-growing cultures (Table 3). Similarly, $10 \mathrm{~min}$ after addition of $10 \mathrm{mM}$ cAMP to a culture of an ethanol-growing $p d e 2$ deletion strain, which is sensitive to externally added cAMP (Wilson et al., 1993), 6PF-2-K activity increased about fourfold (data not shown). No increase in 6PF-2-K activity after glucose addition could be observed in the strain carrying the Pfk $26^{\text {Ala644 }}$ allele (Table 3). However, the basal activity during growth on ethanol was already approximately twofold higher as compared to the wildtype strain. 6PF-2-K activity in the cells containing the Pfk $26^{\text {Asp644 }}$ allele was already very high during growth on ethanol but, surprisingly, was still activatable after glucose addition (Table 3). Activity was even higher in cells containing several copies of the Pfk $26^{\text {Asp644 }}$ allele. Similar results were obtained after addition of cAMP to crude extracts prepared from the different strains grown on ethanol-containing medium (Table 3 ). These results 


\section{Table 3. Activation of wild-type and mutant forms of Pfk26 by glucose or CAMP}

The different strains were grown overnight on a yeast extract/peptone medium with $1 \%$ ethanol to an $\mathrm{OD}_{600}$ of 2-5. 6-PF-2-K activity was determined in crude extracts from these cultures (EtOH) and from the same cultures $10 \mathrm{~min}$ after the addition of $2 \%$ glucose. Additionally, 6PF-2-K activity was determined 5 and $10 \mathrm{~min}$ after the addition of $100 \mu \mathrm{M}$ cAMP to crude extracts prepared after overnight growth on ethanol medium. ND, Not determined. See Table 1 for details of reproducibility.

\begin{tabular}{|c|c|c|c|c|c|}
\hline \multirow[t]{2}{*}{ Strain } & \multirow[t]{2}{*}{ Genotype } & \multicolumn{4}{|c|}{ 6-Phosphofructo-2-kinase activity $\left[\mu \mathrm{U}(\mathrm{mg} \text { protein })^{-1}\right]$} \\
\hline & & $\mathrm{EtOH}$ & $\begin{array}{l}\text { Glucose } \\
\text { (10 min) }\end{array}$ & $\begin{array}{c}\text { cAMP } \\
(5 \mathrm{~min})\end{array}$ & $\begin{array}{l}\text { cAMP } \\
(10 \mathrm{~min})\end{array}$ \\
\hline VW.EB-14B & Pfk $26^{\text {Ser644 }}$ & $4 \cdot 1 \pm 0 \cdot 5$ & $16 \cdot 2 \pm 0 \cdot 5$ & $29 \cdot 4 \pm 1 \cdot 0$ & $29 \cdot 5 \pm 1 \cdot 0$ \\
\hline VW.EB-14C & Pfk26 $6^{\text {Ala644 }}$ & $14 \cdot 0 \pm 2 \cdot 9$ & $9 \cdot 1 \pm 2 \cdot 0$ & $20 \cdot 2 \pm 1 \cdot 9$ & $26 \cdot 2 \pm 1 \cdot 8$ \\
\hline VW.EB-14D & Pfk26Asp644 & $19.5 \pm 0.5$ & $30 \cdot 2 \pm 0.6$ & $39 \cdot 5 \pm 1 \cdot 0$ & $42 \cdot 0 \pm 1 \cdot 4$ \\
\hline VW.EB-14E & {$\left[\mathrm{Pfk} 26^{\mathrm{Asp644}}\right]_{x}$} & $42 \cdot 0 \pm 1 \cdot 6$ & $44 \cdot 2 \pm 1 \cdot 8$ & ND & ND \\
\hline
\end{tabular}

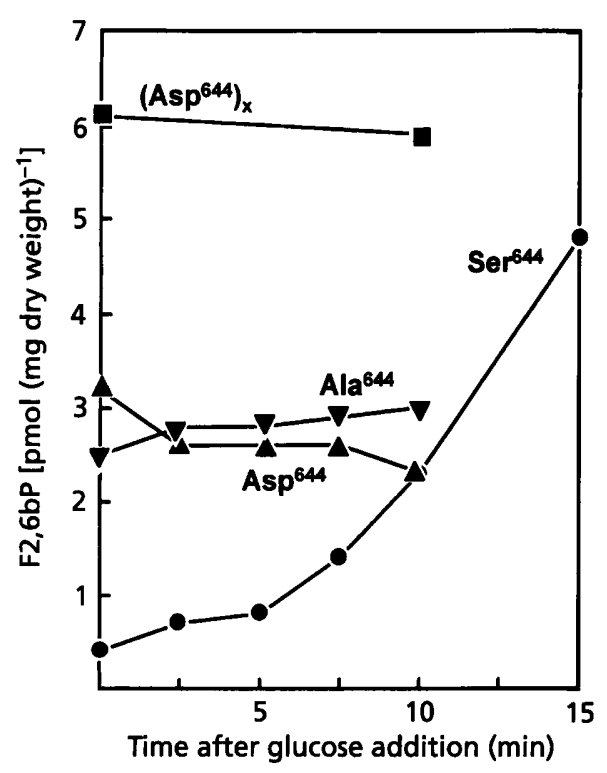

Fig. 1. F2,6bP levels in strains containing the wild-type or

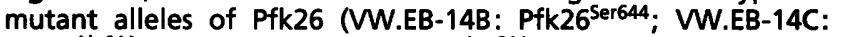

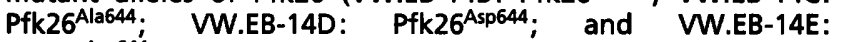
$\left[\text { Pfk26 }{ }^{\text {Asp644 }}\right]_{x}$ ) after overnight growth on a yeast extract/peptone medium with $1 \%$ ethanol and at different times after the addition of $2 \%$ glucose to the cultures.

indicate that serine 644 is involved in the activation of Pfk 26 by protein kinase A phosphorylation. Additionally, the enzyme can be further activated by phosphorylation of another amino acid residue. However, the second activation step is dependent on the efficient phosphorylation of serine 644 .

The different enzymic activities of the mutant Pfk26 alleles were reflected in the levels of F2,6bP after growth on an ethanol medium and after the addition of glucose (Fig. 1). In the wild-type strain the F2,6bP concentration was very low after growth on an ethanol medium but increased to about $5 \mathrm{pmol}(\mathrm{mg} \text { dry weight })^{-1} 15 \mathrm{~min}$ after glucose addition, whereas it had already reached levels of about $6 \mathrm{pmol}(\mathrm{mg} \text { dry weight })^{-1}$ during growth on ethanol in the strain containing several copies of the highly active Pfk $26^{\text {Asp644 }}$ allele. On the other hand, intermediate levels of $\mathrm{F} 2,6 \mathrm{bP}$ were observed in the strains containing one copy of the Pfk $26^{\text {Asp644 }}$ or the Pfk26 ${ }^{\mathrm{Ala644}}$ allele (Fig. 1). Surprisingly, in the case of the strain with one Pfk26 ${ }^{\text {Asp644 }}$ allele, F2,6bP levels decreased after glucose addition although this mutant enzyme is more active than the wild-type form in vitro. This observation suggests that in vivo additional regulatory mechanisms which are also affected by the amino acid exchange might operate on 6PF-2-K activity.

In contrast to our results, it has been reported recently (Keßler \& Eschrich, 1996) that serine 644 is not involved in cAMP-dependent phosphorylation of yeast Pfk 26 but is important for catalytic activity. Keßler \& Eschrich (1996) also used a mutant allele of Pfk 26 that had substituted serine 644 for alanine. We do not understand the reasons for the discrepancies between that and our work. However, in contrast to our work, which uses genomically integrated single copies of the different mutant alleles, the study of Keßler \& Eschrich (1996) used high-copy expression vectors and a nearly thousandfold overexpression of 6PF-2-K enzyme activity, which especially in the case of a tightly regulated enzyme like Pfk26 may cause misleading results.

\section{Increased levels of F2,6bP do not affect F-1,6-bPase activity in vivo}

To test the physiological relevance of inhibition of $\mathrm{F}$ $1,6-\mathrm{bPase}$ by $\mathrm{F} 2,6 \mathrm{bP}$, we investigated the mutant strain containing several copies of the highly active Pfk $26^{\text {Asp644 }}$ allele and exhibiting high concentrations of $\mathrm{F} 2,6 \mathrm{bP}$ [about $6 \mathrm{pmol}$ (mg dry weight) ${ }^{-1}$ ] even under gluconeogenic conditions during growth on ethanol (Fig. 1). From data obtained in vitro (Noda et al., 1984; Marcus et al., 1988) it can be calculated that such high concentrations of F2,6bP should inhibit F-1,6-bPase 
activity nearly completely. However, growth rates of the mutant strain on synthetic or yeast extract/peptone medium with $3 \%$ ethanol as the sole carbon source were identical to those of the wild-type strain. Moreover, we could not observe an increase in the concentration of $\mathrm{F} 1,6 \mathrm{bP}$ or a decrease in the levels of glucose-6-phosphate, F6P or glycogen in the mutant strain as compared to the wild-type strain during growth on a yeast extract/ peptone medium with $3 \%$ ethanol, as would have been expected if F-1,6-bPase was inhibited by the high concentrations of F2,6bP. Therefore, our results indicate that F2,6bP does not inhibit F-1,6-bPase significantly in vivo. This discrepancy from the in vitro measurements might be explained by assuming that other regulatory mechanisms counteract the effect of F2,6bP. Alternatively, it would be tempting to speculate that $F 2,6 \mathrm{bP}$ is not freely available in the cytosol but is restricted to specific compartments (Müller et al., 1996) and therefore is not accessible to F-1,6-bPase in vivo.

On the other hand, it must be concluded from these data that F2,6bP per se is not able to prevent the energywasting futile cycling during the transition from gluconeogenic to fermentative conditions until F-1,6bPase is inactivated and its synthesis is repressed (Entian \& Barnett, 1992). However, it has been shown that inhibition of F-1,6-bPase by F2,6bP is synergistic with that of AMP and is enhanced after phosphorylation of the enzyme (Noda et al., 1984; Marcus et al., 1988). Therefore, it seems that under normal conditions all three inhibitory mechanisms must collaborate to inhibit F-1,6-bPase activity efficiently.

When compared to the wild-type cells, the VW.EB-14E mutant cells containing high concentrations of F2,6bP in an ethanol medium seemed to adapt better to a shift from an ethanol medium to a glucose medium; however, this observation was difficult to quantify. This observation is in accordance with the opposite finding that a strain without F2,6bP exhibited a delay in growth, glucose consumption and ethanol production after such a shift (Boles et al., 1996). During the exponential growth phase in a yeast extract/peptone medium with $2 \%$ glucose, the growth rate of strain VW.EB-14E was identical to that of the wild-type strain $\left(\mu=0.56 \mathrm{~h}^{-1}\right)$. Similarly, glycogen accumulation after nitrogen starvation of this strain was comparable to that of the wildtype strain when incubated in synthetic medium containing $3 \%$ glucose but with no source of nitrogen [13.6 and $13.2 \mu \mathrm{g}(\mathrm{mg} \text { wet weight })^{-1}$, respectively, after $24 \mathrm{~h}$ ].

According to our results, presented here and elsewhere (Boles et al., 1996; Heinisch et al., 1996), it could be suggested that one 6PF-2-K enzyme with a high, constitutive activity should be enough to fulfil all the demands of the yeast cells. Why then did S. cerevisiae develop such an elaborate system for regulation of its F2,6bP concentrations? The answer is difficult but one could imagine that precisely adjustable levels of $F 2,6 \mathrm{bP}$ might be favourable to the cells in specific natural situations which do not occur normally under laboratory conditions or are difficult to quantify.

\section{ACKNOWLEDGEMENTS}

We thank P. Kötter (Frankfurt, Germany) for kindly providing us with strains CEN.PK113-9D and CEN.PK113-3C; M. A. Navas and J. M. Gancedo (Madrid, Spain) for kind provision of pAN11; and M. Kretschmer (Auto-Immune Inc., Lexington, $\mathrm{KY}$, USA) for kind provision of pMK2. We are grateful to E. Schlüter for her excellent technical assistance. This work was supported by the Volkswagen-Stiftung and the European Community (EU contract no. BIO 4-CT95-0107).

\section{REFERENCES}

Arvanitidis, A. \& Heinisch, J. (1994). Studies on the function of yeast phosphofructokinase subunits by in vitro mutagenesis. $J$ Biol Chem 269, 8911-8918.

Bartrons, R., Van Schaftingen, E., Vissers, S. \& Hers, H. G. (1982). Stimulation of yeast phosphofructokinase by fructose-2,6bisphosphate. FEBS Lett 143, 137-140.

Bergmeyer, H. U. (1974). Methods of Enzymatic Analysis. Weinheim: Verlag Chemie.

Boles, E. \& Miosga, T. (1995). A rapid and highly efficient method for PCR-based site-directed mutagenesis using only one new primer. Curr Genet 28, 197-198.

Boles, E. \& Zimmermann, F. K. (1993). Saccharomyces cerevisiae phosphoglucose isomerase and fructose bisphosphate aldolase can be functionally replaced by the corresponding enzymes of Escherichia coli and Drosophila melanogaster. Curr Genet 23, 187-191.

Boles, E., Heinisch, J. \& Zimmermann, F. K. (1993). Different signals control the activation of glycolysis in the yeast Saccharomyces cerevisiae. Yeast 9, 761-770.

Boles, E., Gobhlmann, H. W. H. \& Zimmermann, F. K. (1996). Cloning of a second gene encoding 6-phosphofructo-2-kinase in yeast and characterization of mutant strains without fructose-2,6bisphosphate. Mol Microbiol 20, 65-76.

Breitenbach-Schmitt, I., Heinisch, J., Schmitt, H. D. \& Zimmermann, F. K. (1984). Yeast mutants without phosphofructokinase activity can still perform glycolysis and alcoholic fermentation. Mol Gen Genet 195, 530-535.

Ciriacy, M. \& Breitenbach, I. (1979). Physiological effects of seven different blocks in glycolysis in Saccharomyces cerevisiae. J Bacteriol 139, 152-160.

Davies, S. E. C. \& Brindle, K. M. (1992). Effects of overexpression of phosphofructokinase on glycolysis in the yeast Saccharomyces cerevisiae. Biochemistry 31, 4729-4735.

Entian, K.-D. \& Barnett, J.A. (1992). Regulation of sugar utilization by Saccharomyces cerevisiae. Trends Biochem Sci 17, 506-510.

Fell, D. A. \& Thomas, S. (1995). Physiological control of flux: the requirement for multisite modulation. Biochem J 311, 35-39.

François, J., Van Schaftingen, E. \& Hers, H. G. (1984). The mechanism by which glucose increases fructose-2,6-bisphosphate concentration in Saccharomyces cerevisiae. Eur J Biochem 145, 187-193.

Frančois, J., Eraso, P. \& Gancedo, C. (1987). Changes in the concentrations of cAMP, fructose-2,6-bisphosphate and related metabolites and enzymes in Saccharomyces cerevisiae during growth on glucose. Eur J Biochem 164, 369-373.

François, J., Villanueva, M. E. \& Hers, H. G. (1988). The control of glycogen metabolism in yeast. Eur J Biochem 174, 551-559.

Gancedo, J. M. \& Gancedo, C. (1971). Fructose-1,6-diphosphatase, phosphofructokinase and glucose-6-phosphate dehydrogenase 
from fermenting and non-fermenting yeast. Arch Microbiol 76, 132-138.

Gancedo, J. M., Mazón, M. J. \& Gancedo, C. (1982). Kinetic differences between two interconvertible forms of fructose-1,6bisphosphatase from Saccharomyces cerevisiae. Arch Biochem Biophys 218, 478-482.

Gietz, R. D. \& Sugino, A. (1988). New yeast-Escherichia coli shuttle vectors constructed with in vitro mutagenized yeast genes lacking six base-pair restriction sites. Gene 74, 527-534.

Guthrie, C. \& Fink, E. R. (1991). Guide to yeast genetics and molecular biology. Methods Enzymol 194, 1-933.

Heinisch, J. (1986). Isolation and characterization of the two structural genes coding for phosphofructokinase in yeast. Mol Gen Genet 202, 75-82.

Heinisch, J., Ritzel, R. G., von Borstel, R. C., Aguilera, A., Rodicio, R. \& Zimmermann, F. K. (1989). The phosphofructokinase genes of yeast evolved from two duplication events. Gene 78, 309-321.

Heinisch, J. J., Boles, E. \& Timpel, C. (1996). A yeast phosphofructokinase insensitive to the allosteric activator fructose-2,6bisphosphate. J Biol Chem 271, 15928-15933.

Hers, H. G. \& Van Schaftingen, E. (1982). Fructose-2,6bisphosphate 2 years after its discovery. Biochem J 206, 1-12.

Keßler, R. \& Eschrich, K. (1996). Ser ${ }^{644}$ is important for catalytic activity but is not involved in CAMP-dependent phosphorylation of yeast 6-phosphofructo-2-kinase. FEBS Lett 395, 225-227.

Kretschmer, M. \& Fraenkel, D. G. (1991). Yeast 6-phosphofructo2-kinase: sequence and mutant. Biochemistry 30, 10663-10672.

Kretschmer, M., Tempest, P. \& Fraenkel, D. G. (1991). Identification and cloning of yeast phosphofructokinase 2. Eur J Biochem 197, 367-372.

Lederer, B., Vissers, S., Van Schaftingen, E. \& Hers, H. G. (1981). Fructose-2,6-bisphosphate in yeast. Biochem Biophys Res Commun 103, 1281-1287.

Lillie, S. H. \& Pringle, J. R. (1980). Reserve carbohydrate metabolism in Saccharomyces cerevisiae: responses to nutrient limitation. J Bacteriol 143, 1384-1394.

Marcus, F., Rittenhouse, J., Moberly, L., Edelstein, I., Hiller, E. \& Rogers, D. T. (1988). Yeast (Saccharomyces cerevisiae) fructose1,6-bisphosphatase. J Biol Chem 263, 6058-6062.

Maller, S., Boles, E. \& Zimmermann, F. K. (1996). A two-hybrid system analysis shows interactions between 6-phosphofructo-1kinase and 6-phosphofructo-2-kinase but not between other glycolytic enzymes of the yeast Saccharomyces cerevisiae. Eur J Biochem 236, 626-631.

Navas, M. A. \& Gancedo, J. M. (1996). The regulatory characteristics of yeast fructose-1,6-bisphosphatase confer only a small selective advantage. J Bacteriol 178, 1809-1812.

Navas, M. A., Cerdán, S. \& Gancedo, J. M. (1993). Futile cycles in Saccharomyces cerevisiae strains expressing the gluconeogenic enzymes during growth on glucose. Proc Natl Acad Sci USA 90, 1290-1294.

Noda, T., Hoffschulte, H. \& Holzer, H. (1984). Characterization of fructose-1,6-bisphosphatase from bakers' yeast. J Biol Chem 259, 7191-7197.

Paravicini, G. \& Kretschmer, M. (1992). The yeast FBP26 gene codes for a fructose-2,6-bisphosphatase. Biochemistry 31, 7126-7133.

Sambrook, J., Fritsch, E. F. \& Maniatis, T. (1989). Molecular Cloning: a Laboratory Manual, 2nd edn. Cold Spring Harbor, NY: Cold Spring Harbor Laboratory.

Schaaff, I., Heinisch, J. \& Zimmermann, F. K. (1989). Overproduction of glycolytic enzymes in yeast. Yeast 5, 285-290.

Schiestl, R. H. \& Gietz, R. D. (1989). High efficiency transformation of intact yeast cells by single stranded nucleic acids as carrier. Curr Genet 16, 339-346.

Thevelein, J. M. (1991). Fermentable sugars and intracellular acidification as specific activators of the RAS-adenylate cyclase signalling pathway in yeast: the relationship to nutrient-induced cell cycle control. Mol Microbiol 5, 1301-1307.

Thomas, S. \& Fell, D. A. (1996). Design of metabolic control for large flux changes. $J$ Theor Biol 182, 285-298.

Van Schaftingen, E. (1987). Fructose-2,6-bisphosphate. Adv Enzymol Relat Areas Mol Biol 59, 315-395.

Van Schaftingen, E., Lederer, B., Bartrons, R. \& Hers, H. G. (1982). A kinetic study of pyrophosphate : fructose-6-phosphate phosphotransferase from potato tubers. Eur J Biochem 129, 191-195.

Wilson, R. B., Renault, G., Jacquet, M. \& Tatchell, K. (1993). The pde2 gene of Saccharomyces cerevisiae is allelic to rca1 and encodes a phosphodiesterase which protects the cell from extracellular cAMP. FEBS Lett 325, 191-195.

Zamenhoff, S. (1957). Preparation and assay of deoxyribonucleic acids from animal tissue. Methods Enzymol 3, 696-704.

Received 14 May 1997; accepted 5 June 1997. 